\title{
Go Green- Keep the Root Canal Clean!!!
}

\author{
Zeenath Ambareen, Anitha Chinappa \\ NSVK dental college and hospital, Bangalore \\ Received October 17, 2014; Revised October 18, 2014; Accepted December 18, 2014
}

\begin{abstract}
Disinfecting the root canal system is one of the major objectives in endodontics. Although cleaning and shaping and use of antimicrobial medicaments are effective in reducing the bacterial load, some bacteria persist and multiply, causing reinfection. To counter the ineffectiveness, potential side effects and safety concerns of synthetic drugs, herbal alternatives have been introduced. Herbal irrigants are proven to be safe, contain active constituents that have beneficial physiologic effects. Its curative properties such as antioxidant, anti-inflammatory and radical scavenging activity have proved an added advantage over the traditional root canal irrigants. The use of herbal extracts as endodontic irrigants might be beneficial as a part of a growing trend to seek natural remedies for dental treatment.
\end{abstract}

\section{Keywords: disinfection, endodontics, phytotherapy}

Cite This Article: Zeenath Ambareen, and Anitha Chinappa, “Go Green- Keep the Root Canal Clean!!!” International Journal of Dental Sciences and Research, vol. 2, no. 6B (2014): 21-25. doi: 10.12691/ijdsr-2-6B-7.

\section{Introduction}

"Let food be your medicine and let medicine be your food" was advised by the father of medicine, Hippocrates, over two millennia ago. It's still true today that "you are what you eat." Folk medicine in different cultures has a long history of ancestors creating primitive medicines during their struggles against natural calamity and disease [1]. Many plants with biological and antimicrbiological properties have been studied since there has been a relevant increase in the incidence of antibiotic overuse and misuse. In dentistry Phytomedicines have been used as anti-inflammatory, antibiotic, analgesic and sedative agents [2].

The use of chemical agents during instrumentation to completely clean all aspects of the root canal system is central to successful endodontic treatment [3]. Among the procedure involved in the control of endodontic infection, irrigation is an important step in eliminating microorganisms from the root canal system. Sodium hypochlorite has been the most widely used root canal irrigating solution. The main advantages of sodium hypochlorite are its ability to dissolve necrotic tissues and its antibacterial properties against most microorganisms. However it has several undesirable characteristics such as tissue toxicity, risk of emphysema when overfilling, allergic potential, and disagreeable smell and taste [4]. The constant increase in antibiotic resistant strains and side effects caused by synthetic drugs has prompted researchers to look for herbal alternatives in endodontics. This paper brings to the forefront the undiscovered potential of our traditional herbs.

In dentistry they are used as [5]

1. Antimicrobial: Matricaria chamomile (German chamomile), Salvadora percica (Meswak), Azadirachta indica (Neem).
2. Anti-inflammatory: Plumeria acuminate (Champa), Kalanchoe Brasiliensis (Air plant), Mikania cordifolia (Guaco), Propolis.

3. Sedative and Anxiolytics: Melissa officinalis (Lemon balm), Passiflora incarnale (Passion Flower), Piper meythsticum.

4. Miscellaneous: Endodontic irrigants, medicaments and endodontic retreatment.

\section{Morinda Citrofolia [MC]}

Among the medicinal plants discovered by the ancestors of Polynesians, Morinda citrifolia [Noni] is one of the traditional folk medicinal plants that has been used for over 2000 years in Polynesia [1]. Noni is the common name for Morinda citrifolia and is also called Indian Mulberry, Ba Ji Tian, Nono or Nonu, Cheese Fruit, and Nhau in various cultures throughout the world.

It has been reported to have a broad range of therapeutic and nutritional value. The Polynesians utilized the whole Noni plant in various combinations for herbal remedies. The fruit juice is in high demand in alternative medicine for different kinds of illnesses such as arthritis, diabetes, high blood pressure, muscle aches and pains, menstrual difficulties, headaches, heart disease, AIDS, cancers, gastric ulcers, sprains, mental depression, senility, poor digestion, atherosclerosis, blood vessel problems, and drug addiction.

A number of major components have been identified in the Noni plant such as scopoletin, octoanoic acid, potassium, vitamin $\mathrm{C}$, terpenoids, alkaloids, anthraquinones (such as nordamnacanthal, morindone, rubiadin, and rubiadin- 1methyl ether, anthraquinone glycoside), b-sitosterol, carotene, vitamin A, flavone glycosides, linoleic acid, Alizarin, amino acids, acubin, $L$-asperuloside, caproic acid, caprylic acid, ursolic acid, rutin, and a putative proxeronine. 
Acubin, L-asperuloside, and alizarin in the Noni fruit, as well as some other anthraquinone compounds in Noni roots, are all proven antibacterial agents. These compounds have been shown to fight against infectious bacteria strains such as Pseudomonas aeruginosa, Proteus morgaii, Staphylococcus aureus, Baciillis subtilis, Escherichia coli, Salmonella, and Shigela.

An in vitro study compared the effectiveness of the juice of $\mathrm{MC}$ with $\mathrm{NaOCl}$ and Chlorhexidine to remove the smear layer from root canal walls of instrumented teeth. It was concluded that the efficacy of Morinda Citrifolia was similar to $\mathrm{NaOCl}$ in conjunction with EDTA as an intracanal irrigant [5].

The antimicrobial activity of 2\% CHX gel, propolis, Morinda Citrifolia juice and calcium hydroxide has been compared on E.faecalis infected root canal dentin at two different depths and three intervals. It was concluded that Propolis and Morinda Citrifolia were effective against E. faecalis in dentin on extracted teeth [6].

Morinda Citrifolia appears to be the first juice to be identified as a possible alternative to the use of $\mathrm{NaOCl}$ as an intracanal irrigant.

\section{Neem}

Azadirachta indica A. Juss is a commonly seen medicinal tree in India, which is considered holy. Popularly known as "Indian neem/ Margosa tree" or "Indian lilac", is well known in India and its neighboring countries for more than 2000 years as one of the most versatile medicinal plants having a wide spectrum of biological activity. In Sanskrit, it is called "arishtha" meaning "reliever of sickness" and is regarded as the village dispensary of India. Importance of neem tree has been recognized by US National Academy of Sciences where neem is entitled as 'a tree for solving global problems' [7].

Various parts of the neem tree have been used as traditional ayurvedic medicine in India from time immemorial. The medicinal utilities have been described, especially for leaf, fruit and bark. Neem oil and the bark and leaf extracts have been therapeutically used as folk medicine to control leprosy, intestinal helminthiasis, respiratory disorders, constipation and also as a general health promoter. Its use for the treatment of rheumatism, chronic syphilitic sores and indolent ulcer has also been evident. Neem oil finds use to control various skin infections. Bark, leaf, root, flower and fruit together cure blood morbidity, biliary afflictions, itching, skin ulcers, burning sensations and pthysis [8].

Biologically active principles isolated from different parts of the plant include: azadirachtin, meliacin, gedunin, salanin, nimbin, valassin and many other derivatives of these principles. Miliacin forms the bitter principles of neem seed oil (NSO), the seed also contain tignic acid (5methyl- 2-butanic acid) responsible for the distinctive odor of the oil.

The compounds have been divided into two major classes: isoprenoids and others. The isoprenoids include diterpenoids and triterpenoids containing protomeliacins, limonoids, azadirone and its derivatives, gedunin and its derivatives, vilasinin type of compounds and csecomeliacins such as nimbin, salanin and azadirachtin. The nonisoprenoids include proteins (amino acids) and carbohydrates (polysaccharides), sulphurous compounds, polyphenolics such as flavonoids and their glycosides, dihydrochalcone, coumarin and tannins, aliphatic compounds, etc.

Although a large number of compounds have been isolated from various parts of neem, Nimbidin, a major crude bitter principle extracted from the oil of seed kernels of A. indica demonstrated antiinflammatory, antiarthritic, antipyretic, hypoglycaemic, antigastric ulcer, spermicidal, antifungal, antibacterial, diuretic properties. From this crude principle some tetranortriterpenes, including nimbin, nimbinin, nimbidinin, nimbolide and nimbidic acid have been isolated.

Oil from the leaves, seeds and bark possesses a wide spectrum of antibacterial action against Gram-negative and Gram-positive microorganisms, including $\mathrm{M}$. tuberculosis and streptomycin resistant strains. In vitro, it inhibits Vibrio cholerae, Klebsiella pneumoniae, $\mathrm{M}$. tuberculosis and M. pyogenes. Antimicrobial effects of neem extract have been demonstrated against Streptococcus mutans and E. faecalis [8].

Neem also has an anti-adherence activity by altering bacterial adhesion and ability of organism to colonize [9]. It has been shown that neem is highly effective in the treatment of periodontal disease. Its biocompatibility to human periodontal ligament fibroblasts is an important factor favoring its clinical application [10].

Use of neem as an endodontic irrigant might be advantageous because it is a biocompatible antioxidant and thus not likely to cause the severe injuries to patients that might occur via $\mathrm{NaOCl}$ accidents. Bitter taste associated with this plant can be altered by different formulations due to addition of sweeteners and flavors to increase the patient compliance and acceptability [11].

A study showed significant differences in the zone of inhibition of diameters of neem extract and $2 \% \mathrm{NaOCl}$ against E.faecalis and mixed culture [12].

\section{Propolis}

The word Propolis is believed to have been coined by Aristotle who identified how propolis was used to protect and defend the hive. Propolis (from the Greek) means 'Before the City' or 'Defender of the City'. In the beginning of the Greek civilization, Aristotle observed that propolis had the ability to defend a city with thousands of inhabitants, the beehive.

Propolis is resinous material/sap that is collected after it oozes out from tree bark and bud. After bees have collected propolis they mix it with wax flakes and their saliva in the hive. This mixture is what they use to cover the interior of the hive. The bees not only use propolis as a building material and structural defence mechanism but their health is maintained as a result of its immune system enhancing properties. Propolis forms the bees external immune defence system, making the beehive one of the most sterile environments known to nature [13].

Propolis is a resin widely used in folk medicine for centuries. The Egyptians used propolis, honey and other resins to mummify their pharaohs preserving them, as far as possible, for the next life. Propolis has been used by man since these times in one form or another to stay well and to treat disease. It is known that propolis exhibits 
several pharmacological properties such as antimicrobial, anti-inflammatory, healing, anesthetic, cytostatic and cariostatic properties. In China, propoils was authorized as a new material medicine and embodied in the Chinese pharmacopeia in 2005.

In Dentistry, propolis has been used for the reatment of aphthous ulcers, Candida albicans, acute necrotizing ulcerative gingivitis [ANUG], gingivitis and periodontitis, and recently as a storage medium for teeth that get avulsed from its socket, in order to maintain the viability of the periodontal ligament cells. The anti-inflammatory property of propolis is due to the presence of caffeic acid and phenethyl ester [CAPE] in propolis. Ethanol extract of propolis presents good properties for endodontic use, such as promoting bone regeneration and inducing hard tissue bridge formation in pulpotomies or pulp capping. Propolis is dispensed in various forms. Propolis being a good antimicrobial and anti-inflammatory agent, can serve as a better intracanal irrigant and intracanal medicament.

It is composed of resin and balsams (50 - 70\%), essential oils and wax (30 - 50\%), pollen (5 - 10\%) and other constituents which are amino acids, minerals, vitamins A, B complex, E and the highly active biochemical substance known as bioflavonoids, phenols and aromatic compounds [15].

Flavonoids and cinnamic acid derivatives have been considered as the main primary biologically active components. Flavonoids, are all ketone-containing compounds, and are the most important plant pigments for flower coloration producing yellow or red/blue pigmentation in petals designed to attract pollinator animals. Flavonoids are the most common group of polyphenolic compounds in the human diet and are found ubiquitously in plants. They are divided into four subgroups: Flavones, Flavonol, Flavonones, Flavononol [13].

The antimicrobial properties of propolis have been attributed to its high flavonoid content and in particular the presence of the flavonoids galangin and pinocembrin. This herbal medicine has been used systemically and topically for thousands of years in China for the treatment of periodontal abscesses and infected oral wounds. The flavone baicalein is reported to be largely responsible for antimicrobial effects [16].

The antibacterial efficacy of three commonly used intracanal medicaments with propolis against Enterococcus faecalis has been compared. They concluded that propolis had good invitro antibacterial activity against Enterococcus faecalis in the root canals, suggesting that it could be used as an alternative intracanal medicament. The ntimicrobial activity of propolis with calcium hydroxide as intracanal, medicament against Enterococcus faecalis found that propolis was effective in eliminating the microorganisms [6].

Al-Qathami and Al-Madi (2003) compared the antimicrobial efficacy of propolis, sodium hypochlorite and saline as an intracanal irrigants. Microbiological samples were taken from the teeth immediately after accessing the canal and after instrumentation and irrigation. The results of this study indicated that the propolis has antimicrobial activity equal to that of sodium hypochlorite [17].

\section{Tea Tree Oil}

Tea tree oil is extracted from the tree Melaleuca alternifolia that grows in Australia, and has been shown to have many beneficial medicinal uses as an antiseptic, antifungal and antibacterial agent. Studies indicate that Melaleuca alternifolia is extracted from the leaves and twigs by steam distillation and the yield is about $1.8 \%$ and that the main chemical component to have antimicrobial activity in tea tree oil is attributed to terpinen-4-ol. It has also been used to treat cuts, minor burns, athlete's foot, and insect bites. Studies reveals that it can treat bacterial and fungal skin infections, wound infections, gum infections, acne, head lice, eczema, vaginal yeast infections, pneumonia, and other respiratory illnesses.

It also has mild solvent action, and hence could hold potential applications in root canal treatment for dissolving the necrotic pulp tissue. This compound is responsible for its antibacterial and antifungal properties [18].

Groppo FC et al conducted a study based upon the comparision of the anti microbial activity of garlic, Tea tree oil, and chlorhexidine against oral micro organisms. His study revealed that garlic and chlorhexidine showed good anti microbial activity against Streptococcus mutans than other oral micro organisms, whereas, TTO showed significant antimicrobial effect against Streptococcus mutans and other oral micro organisms [19].

An in vitro study showed that tea tree oil might disinfect the root canal system as effectively as $\mathrm{NaOCl}$. Further, the toxicity of tea tree oil is lesser than $\mathrm{NaOCl}$ [20].

\section{Green Tea Polyphenols}

Green tea (GT), extracted from Camellia sinensis, is a widely consumed beverage throughout the world that is second only to water. Green tea extracts (GTEs) contain catechin, which is one of the polyphenols from GT. Catechins in GT are catechin, epicatechin, epicatechin gallate, epigallocatechin, epigallocatechin-3-gallate (EGCG), and so forth. It has been reported that GTEs have remarkable antiinflammatory, antioxidant, and anticarcinogenic effects in a number of animal tumors, cell culture systems, and epidemiological studies [22]. A recent animal study showed that the GTE in combination with low-dose cyclosporine A prolonged allograft survivals in mice [23].

In the dental field, the usage of GTEs has been still studied. EGCG was known to protect the alveolar bone resorption from periodontal diseases because it inhibited the expression of matrix metalloproteinase-9 (MMP-9) in osteoblasts and the formation of osteoclasts [24]. Also, EGCG suppressed the progression of apical periodontitis, possibly by diminishing osteobalstic expression of cysteinerich and subsequently macrophage chemotaxis into the lesions in rats [25].

An in vitro study conducted to evaluate the antimicrobial efficacy of Triphala, GTPs, MTAD, and 5\% Sodium Hypochlorite against E-faecalis biofilm formed on tooth substrate showed maximum antibacterial activity with $\mathrm{NaOCl}$ and statistically significant antibacterial activity with Triphala, GTPs and MTAD [22].

\section{Triphala}

Triphala is an Indian ayurvedic herbal formulation consisting of dried and powdered fruits of three medicinal 
plants Terminalia bellerica, Terminalia chebula, and Emblica officinalis. Triphala has been proven to be safe, containing active constituents that have beneficial physiologic effect apart from its curative property such as antioxidant, antiinflammatory, and radical scavenging activity [26]. Its fruit is rich in citric acid, which may aid in removal of smear layer thereby acting as chelating agent and also found to be alternative to sodium hypochlorite for root canal irrigation.

A recent study showed that Triphala was as effective as $\mathrm{NaOCl}$ and a doxycycline based irrigant on root canal biofilms that were 3 weeks old. It brought a reduction in E.fecalis counts, when compared to saline. Moreover, Triphala is also a very good chelating agent because of the fruits that are rich in citric acid, and holds promise in the removal of smear layer [22].

\section{Garlic}

Allium sativum, commonly known as garlic, is a species in the onion genus, Allium. It has been used throughout its history for both culinary and medicinal purposes. The garlic

plant's bulb is the most commonly used part. Garlic cloves are used for consumption or for medicinal purposes. In vitro studies indicate that garlic has been found to have antibacterial, antiviral, and antifungal activity. Allium sativum has been found to reduce platelet aggregation and hyperlipidemia. It is also an anti-diabetic agent.

Allium sativum yields allicin, an antibiotic and antifungal compound (phytoncide). It can be used as a home remedy to help speed recovery from sore throat or other minor ailments because of its antibiotic properties. It also contains the sulfur-containing compounds alliin, ajoene, diallylsulfide, dithiin, S-allylcysteine, and enzymes, B vitamins, proteins, minerals, saponins, flavonoids, and Maillard reaction products, which are not sulfur-containing compounds. Furthermore, a phytoalexin (allixin) was found, a nonsulfur compound with a $\gamma$ pyrone skeleton structure with antioxidant, antimicrobial effects, antitumor promoting effects, inhibition of aflatoxin B2 DNA binding, and neurotrophic effects.

Garlic possess diaphoretic, expectorant, antispasmodic, antiseptic, bacteriostatic, antiviral, antihelminthic and hypotensive effects; it is commonly used to treat chronic bronchitis, recurrent upper respiratory tract infections and influenza. In Europe and India, garlic remedies are used to treat coughs, colds, hay fever and asthma [26].

The main active component of garlic is allicin. It is antibacterial and has immune regulatory functions. Allicin destroys cell wall and cell membrane of root canal bacteria [26]. This is used as an alternative irrigant to $\mathrm{NaOCl}$. Garlic extract inhibits the growth of oral pathogens like Streptococcus mutans and Porphyromonas gingivalis hence used for management of dental infections like periodontitis [27].

\section{Conclusion}

The studies done so far are revolutionary because they suggest that intracanal irrigants and dental disinfecting solutions can be formulated from herbs. Though research in this area has barely begun, it will not be surprising if research into the millions of compounds contained in fruit juices, seeds, roots, bark, and flowers will identify further compounds with the potential to be used in dentistry and endodontics. In this era of evidence based medicine, any material with potential clinical applications must go through a series of tests to demonstrate biocompatibility to the tissues of the oral cavity as well as marked advantages in terms of efficacy in root canal disinfection, when compared to contemporary irrigants.

\section{References}

[1] Wang MY, West BJ, Jensen CJ, et al. Morinda citrifolia (Noni): a literature review and recent advances in Noni research. Acta Pharmacol Sin 2002:23; 1127-41.

[2] Sharad Kamat, Rajeev K, Prahlad Saraf. Role of herbs in endodontics: An update. Endodontology 2011; 23: 98-102.

[3] Y. Shen, S. Stojicic, W. Qian, I. Olsen, and M. Haapasalo, “The synergistic antimicrobial effect by mechanical agitation and two chlorhexidine preparations on biofilm bacteria," Journal of Endodontics, vol. 36, no. 1, pp. 100-104, 2010.

[4] Segura JJ, Jimenez-Rubio A, Guerrero JM, Calvo JR. Comparative effects of two endodontic irrigants, chlorhexidine digluconate and sodium hypochlorite on macrophage adhesion to plastic surface. J Endod 1999; 25 : 243-46.(ginger)

[5] Murray PE, Farber RM, Namerow KN, Kuttler S, Garcia Godoy F. Evaluation of Morinda citrofolia as an endodontic irrigant. J Endod 2008; 34: 66 -70.

[6] Kandaswamy D, Venkatesh babu N, Gogulnath D, Kindo AJ. Dentinal tubule disinfection with $2 \%$ chlorexidine gel, propolis, Morinda citrifolia juice, $2 \%$ povidine iodine and calcium hydroxide. Int Endod J 2010; 43: 419-423.

[7] Biswas K, Chattopadhyay I, Banerjee RK, Bandyopadhyay U. Biological activities and medicinal properties of neem [Azadirachta indica] Current Science 2002; 82: 1336-1345.

[8] P. Sudhir Kumar, Debasis Mishra, Goutam Ghos and Chandra S. Panda. Biological action and medicinal properties of various constituent of Azadirachta indica (Meliaceae)" an Overview. Annals of Biological Research, 2010, 1 (3):24-34.

[9] Pereira J, Bergamo D, Franca S, Pietro R, Silva- Sousa Y, Antimicrobial activity of articum lappa against microorganisms commonly found in endodontic infections, Braz Dent J 2005; 16[3]: 192-196.

[10] Behl H, Sidhu O, Kumar V, Singh D, Saimbi C. Efficacy of neem active metabolites for prevention of dental plaque and gingivitis, Neem Foundation 2002.

[11] Botelho M, Araujo Dos Santos, Martins J, Carvalho C, Paz M, Azenha C, Ruela R, Queiroz D, Ruela W, Marino G, Ruela F, Efficacy of a mouthrinse based on leaves of neem in the treatment of patients with chronic gingivitis, J Medicinal Plants Research 2008; 2: 341-346.

[12] Bohora A, Hegde V, Kokate S. Comparison of the antibacterial efficiency of neem leaf extract and $2 \%$ sodium hypochlorite against E. faecalis, C. albicans and mixed culture - An in vitro study. Endodontology 2010; 22: 8-12.

[13] V Ahuja, A Ahuja. Apitherapy - A sweet approach to dental diseases. Part II: Propolis. Journal of Academy of Advanced Dental Research, Vol 2; Issue 2: May 2011.

[14] Da Silva F B, De Almeida J M, De Sousa S M G. Natural medicaments in endodontics - a comparative study of the antiinflammatory action. Braz Oral Res 2004; 18(2):174-9.

[15] Abhishek Parolia, Manuel S. Thomas, M. Kundabala and Mandakini Mohan. Propolis and its potential uses in oral health. International Journal of Medicine and Medical Sciences Vol. 2(7) pp. 210-215, July 2010.

[16] T.P. Tim Cushnie, Andrew J. Lamb. Antimicrobial activity of flavonoids. International Journal of Antimicrobial Agents 26 (2005) 343-356.

[17] Hind Al-Qathami, Ebtissam Al-Madi. Comparison of sodium hypochlorite, propolis and saline as root canal irrigants: A pilot study. Saudi Dental Journal, Vol. 15, No. 2, May - August 2003.

[18] Dhinahar.S, Lakshmi.T. role of botanicals as antimicrobial agents in management of dental infections - A Review. International Journal of Pharma and Bio Sciences. Vol 2, Issue 4, Oct-Dec 2011. 
[19] Groppo FC, Ramacciato JC, Simoes RP, Florio FM, Sartoratto A Antimicrobial activity of garlic, tea tree oil, and chlorhexidine against oral microorganisms. Int Dent J. 2002 Dec; 52(6):433-7.

[20] Sader Lahijini, Raoof kateb, R,Yazdini D. The effect of German chamomile (Marticaria recutita L.) extract and tea tree (Melaleuca alternifolia L.) oil used as irrigants: a scanning electron microscope study. Int Endod J 2006: 39: 190-195.

[21] Ji Young Hwang, Sung Chul Choi, Jae-Hong Park, Sang Wook Kang. The Use of Green Tea Extract as a Storage Medium for the Avulsed Tooth. Journal of Endodontics Volume 37, Number 7, July 2011.

[22] Prabhakar J, Senthilkumar M, Priya MS, Mahalakshmi K, Sehgal PK, Sukumaran VG. Evaluation of antimicrobial efficacy of herbal alternatives (Triphala and green tea polyphenols), MTAD, and 5\% sodium hypochlorite against Enterococcus faecalis biofilm formed on tooth substrate: an in vitro study. J Endod 2010; 36: 836.
[23] Tripathi S, Bruch D, Gatto LA, Kittur DS. Green tea extract prolongs allograft survival as an adjunctive therapy along with low dose cyclosporine A. J Surg Res 2009; 154: 85-90.

[24] Yun JH, Pang EK, Kim CS, et al. Inhibitory effects of green tea polyphenol (-)-epigallocatechin gallate on the expression of matrix metalloproteinase-9 and on the formation of osteoclasts. J Periodontal Res 2004; 39: 300-7.

[25] Lee YL, Hong CY, Kok SH, et al. An extract of green tea, epigallocatechin-3-gallate, reduces periapical lesions by inhibiting cysteine-rich 61 expression in osteoblasts. J Endod 2009; 35: 20611.

[26] Prasanna Neelakantan, Nithya Jagannathan, Nabeel Nazar Ethnopharmacological approach in Endodontic Treatment: A Focused Review. Int. J. Drug Dev. \& Res., Oct-Dec 2011, 3 (4): 68-77.

[27] I.M Bakri and C.W.I. Dowglas: Inhibitory effect of garlic extract on oral bacteria Archives of Oral Biology Volume 50, Issue 7, July 2005, Pages 645-651. 